\title{
Correction to: Mucormycosis in hospitalized patients at a tertiary care center in Lebanon: a case series
}

\author{
Saeed El Zein ${ }^{1} \cdot$ Jean El-Cheikh ${ }^{2} \cdot$ Aline El Zakhem $^{1} \cdot$ Dima Ibrahim $^{1} \cdot$ Ali Bazarbachi $^{2} \cdot$ Souha S. Kanj $^{1}$
}

Published online: 7 January 2019

○) Springer-Verlag GmbH Germany, part of Springer Nature 2019

Correction to: Infection (2018) 46:811-821

https://doi.org/10.1007/s15010-018-1195-4

Unfortunately, the original version of this article contains mistakes. The names "Jean El-Cheikh" and Aline El Zakhem were spelled incorrectly.

In addition, a further correction has been published previously to correct the spelling of the name "Ali Bazarbachi".

The original article can be found online at https://doi.org/10.1007/ s15010-018-1195-4.

Souha S. Kanj

sk11@aub.edu.lb

1 Division of Infectious Diseases, American University

of Beirut Medical Center, Riad El Solh, PO Box 11-0236,

Beirut 1107 2020, Lebanon

2 Division of Hematology Oncology, Department of Internal Medicine, American University of Beirut Medical Center, Beirut, Lebanon 